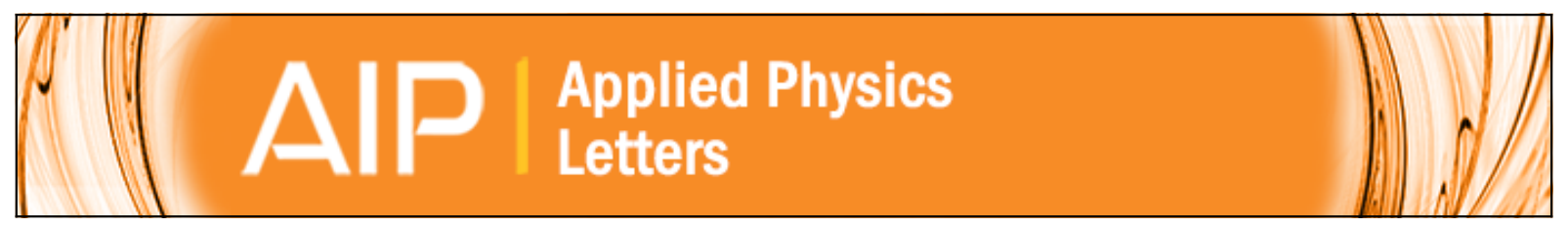

Microstructures of coherent phase decomposition near morphotropic phase boundary in lead zirconate titanate

Wei-Feng Rao and Yu U. Wang

Citation: Applied Physics Letters 91, 052901 (2007); doi: 10.1063/1.2767146

View online: http://dx.doi.org/10.1063/1.2767146

View Table of Contents: http://scitation.aip.org/content/aip/journal/apl/91/5?ver=pdfcov

Published by the AIP Publishing

Over 700 papers \&

presentations on

multiphysics simulation visw now

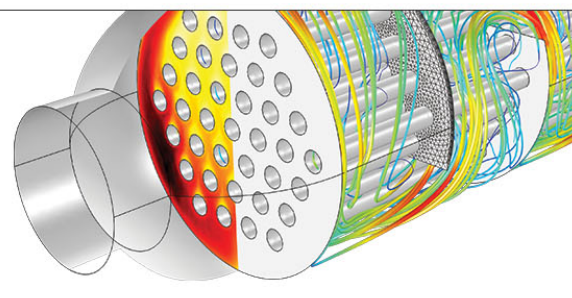




\title{
Microstructures of coherent phase decomposition near morphotropic phase boundary in lead zirconate titanate
}

\author{
Wei-Feng Rao and Yu U. Wang ${ }^{\text {a) }}$ \\ Department of Materials Science and Engineering, Virginia Tech, Blacksburg, Virginia 24061
}

(Received 10 May 2007; accepted 23 May 2007; published online 30 July 2007)

\begin{abstract}
Microstructures of coherent phase decomposition near morphotropic phase boundary in lead zirconate titanate are investigated by using computer modeling and simulation. The model is based on the recently proposed incoherent equilibrium phase diagram [G. A. Rossetti, Jr. et al., Appl. Phys. Lett. 88, 072912 (2006)] and takes into account the coherency strain energy and electrostatic energy. It reveals characteristic multidomain microstructures, where nanoscale lamellar domains of tetragonal and rhombohedral phases coexist with well-defined crystallographic orientation relationships and produce coherent diffraction effects. The simulated microstructures provide important information for interpretation of diffraction data and identification of phases near morphotropic phase boundary. ( 2007 American Institute of Physics. [DOI: 10.1063/1.2767146]
\end{abstract}

Phase coexistence is commonly observed near the morphotropic phase boundary (MPB) in lead zirconate titanate, $\mathrm{Pb}\left(\mathrm{Zr}_{1-x} \mathrm{Ti}_{x}\right) \mathrm{O}_{3}(\mathrm{PZT}){ }^{1}$ The MPB is a nearly vertical phase boundary in the temperature-composition phase diagram, which separates rhombohedral and tetragonal ferroelectric phases at low and high Ti contents, respectively. The presence of MPB as a sharp line phase boundary violates the Gibbs phase rule of equilibrium thermodynamics. ${ }^{2}$ The origin of phase coexistence has been a topic of many investigations $^{2-7}$ and remains as an open question. Recently, a new intermediate monoclinic phase was reported based on $\mathrm{X}$-ray diffraction experiment, ${ }^{8}$ which renews research interest in PZT phase diagram.

The phases in PZT system are usually identified by x-ray or neutron powder diffractions. When the microstructure size is much smaller than the coherence length of diffraction radiations, i.e., nanoscale microstructures, scattered waves from individual nanodomains coherently superimpose in diffraction, where the resultant diffraction intensity profiles only reflect the average diffraction effect of crystal lattices over multiple nanodomains. Recent nanotwin diffraction analysis shows that nanotwins of tetragonal and rhombohedral phases are perceived by x-ray and neutron diffractions as monoclinic phases, where adaptive Bragg reflection peaks appear between the conventional twin peaks of coarse domains, and the positions of the new peaks are determined by the relative thicknesses of nanotwin variants. ${ }^{9}$ Such extraordinary diffraction peaks cannot be explained by conventional diffraction theory of coarse domains, and an introduction of monoclinic phase is found to improve the Rietveld refinement of diffraction data, which leads to the report of new monoclinic phase. ${ }^{8}$ It is noteworthy that such an adaptive diffraction phenomenon from nanodomain microstructures has been directly observed in PZT near MPB by recent complementary $\mathrm{X}$-ray powder diffraction and transmission electron microscopy experiments, ${ }^{10}$ where the appearance of a new peak previously linked to monoclinic phase is directly correlated to the formation of nanodomains. Without taking into account the nanoscale domain microstructures, the dif-

${ }^{a)}$ Author to whom correspondence should be addressed; electronic mail: yuwang@mse.vt.edu fraction data have been misinterpreted and led to identification of wrong phase. In order to correctly interpret diffraction data and identify PZT phases, it is crucial to know the nanodomain microstructures. The purpose of this letter is to report characteristic two-phase multidomain microstructures that are formed by phase decomposition near MPB, gain insight into nanodomain effects on diffraction, and better understand the phase behavior and phase diagram of PZT.

It has been long-recognized that a two-phase zone is required around MPB, and the MPB is considered as located at the composition where the two phases coexist in equal quantity. ${ }^{1}$ Recently, an extended thermodynamic theory of PZT solid solution was formulated, ${ }^{2}$ which treats PZT system as a regular solution and combines the conventional Landau-Ginzburg-Devonshire (LGD) free energy function ${ }^{11}$ with positive enthalpy of formation ${ }^{12}$ and entropy of mixing. The new free energy formulation automatically predicts equilibrium (diffusional) PZT phase diagram with two-phase region replacing the linear MPB and satisfying the Gibbs phase rule. ${ }^{2}$ In particular, it predicts phase decomposition through peritectoid reaction, where diffusion rate is estimated to be sufficiently fast during normal material processing. ${ }^{2}$ It is worth noting, while providing valuable insight into the phase coexistence phenomenon, the predicted phase diagram is for incoherent phases, i.e., coherency strain energy is not considered. In real PZT samples, coherent phases are expected because of the limited dislocation activities and nanoscale decomposition microstructures. In this work, we employ computer modeling and simulation to study the effects of coherency strain energy (and electrostatic energy and polarization gradient energy as well) on domain microstructure evolution during phase decomposition near the MPB in PZT.

Phase-decomposing ferroelectric PZT is a compositionally, structurally, and electrically heterogeneous system with multiphase and multidomain microstructures, whose state is described by the composition (molar fraction) scalar field $x(\mathbf{r})$ and polarization vector field $\mathbf{P}(\mathbf{r})$. The total system free energy is ${ }^{13-15}$ 


$$
\begin{aligned}
F= & \int d^{3} r\left[f(x, \mathbf{P})+\frac{1}{2} \beta_{i j k l}^{\mathbf{P}} \nabla_{i} P_{j} \nabla_{k} P_{l}+\frac{1}{2} \beta_{i j}^{x} \nabla_{i} x \nabla_{j} x\right] \\
& +\frac{1}{2} \int \frac{d^{3} k}{(2 \pi)^{3}}\left[\frac{n_{i} n_{j}}{\varepsilon_{0}} \widetilde{P}_{i} \widetilde{P}_{j}^{*}+K_{i j k l} \widetilde{\varepsilon}_{i j}^{0} \widetilde{\varepsilon}_{k l}^{* *}\right],
\end{aligned}
$$

where summation convention over repeated indices is implied. The function $f(x, \mathbf{P})$ is the nonequilibrium local bulk free energy density that defines the thermodynamic properties of ferroelectric phases in stress-free homogeneous states. We adopt the recently proposed free energy formulation, ${ }^{2}$

$$
\begin{aligned}
f(x, \mathbf{P})= & \frac{a_{2}(x)}{2}\left[T-T_{C}(x)\right]\left(P_{1}^{2}+P_{2}^{2}+P_{3}^{2}\right)+\frac{a_{4}}{4}\left(x-x_{m}\right) \\
& \times\left(P_{1}^{2}+P_{2}^{2}+P_{3}^{2}\right)^{2}+\frac{a_{4}^{\prime}}{4}\left(x-x_{m}\right)\left(P_{1}^{4}+P_{2}^{4}+P_{3}^{4}\right) \\
& +\frac{a_{6}}{6}\left(P_{1}^{2}+P_{2}^{2}+P_{3}^{2}\right)^{3}+\frac{w}{2} x(1-x) \\
& +\frac{k_{B} T}{\Omega}[x \ln x+(1-x) \ln (1-x)],
\end{aligned}
$$

where $T$ is temperature, $T_{C}$ is Curie-Weiss temperature of $\mathrm{PbTiO}_{3}, x_{m}$ is the MPB composition, $w$ is the atomic exchange interaction parameter characterizing enthalpy of mixing, $k_{B}$ is Boltzmann's constant, $\Omega$ is the unit cell volume of $\mathrm{PbTiO}_{3}$, and $a$ 's are the polynomial coefficients of LGD expansion of the polarization-dependent part of the bulk free energy. The first gradient term in Eq. (1) characterizes the energy contribution associated with polarization change across domain walls; the second gradient term, which is absent in compositionally homogeneous (diffusionless) ferroelectrics, ${ }^{13-15}$ characterizes the energy contribution from composition gradient across interfaces between phases of different compositions. The tensors $\beta_{i j k l}^{\mathbf{P}}$ and $\beta_{i j}^{x}$ are the gradient coefficients, and $\nabla_{i}=\partial / \partial r_{i}$ is gradient operator. The $\mathbf{k}$-space integral in Eq. (1) gives the electrostatic energy of polarization distribution $\mathbf{P}(\mathbf{r})$ and the elastostatic energy of misfit strain distribution $\varepsilon^{0}(\mathbf{r})$, where $\varepsilon_{0}$ is the permittivity of free space, $K_{i j k l}=C_{i j k l}-n_{m} C_{i j m n} \Omega_{n p} C_{k l p q} n_{q}, \Omega_{i k}=\left(C_{i j k l} n_{j} n_{l}\right)^{-1}$, $C_{i j k l}$ is the elastic modulus tensor, and $\mathbf{n}=\mathbf{k} / k$. The spontaneous lattice misfit strain is coupled to polarization through electrostriction coefficient tensor $Q_{i j k l}, \varepsilon_{i j}^{0}=Q_{i j k l} P_{k} P_{l}$. The functions $\widetilde{\mathbf{P}}(\mathbf{k})$ and $\widetilde{\boldsymbol{\varepsilon}}^{0}(\mathbf{k})$ are the Fourier transform of the fields $\mathbf{P}(\mathbf{r})$ and $\varepsilon^{0}(\mathbf{r})$, respectively, and the superscript asterisk ${ }^{*}$ indicates the complex conjugate. The phase decomposition process is characterized by the Cahn-Hilliard nonlinear diffusion equation, ${ }^{16}$

$$
\frac{\partial x(\mathbf{r}, t)}{\partial t}=\nabla \cdot D \nabla \frac{\delta F}{\delta x(\mathbf{r}, t)},
$$

where $D$ is the chemical mobility of diffusion. The evolutions of polarization and domain microstructures are characterized by the time-dependent Ginzburg-Landau equation, ${ }^{13-15}$

$$
\frac{\partial P_{i}(\mathbf{r}, t)}{\partial t}=-L_{i j} \frac{\delta F}{\delta P_{j}(\mathbf{r}, t)},
$$

where $L_{i j}$ is the kinetic coefficient tensor for displacive (diffusionless) evolution of polarization. In this work, we use $\beta_{i j k l}^{\mathbf{P}}=\beta^{\mathrm{P}} \delta_{i k} \delta_{j l}, \beta_{i j}^{x}=\beta^{x} \delta_{i j}, L_{i j}=L \delta_{i j}$, and the reported bulk free
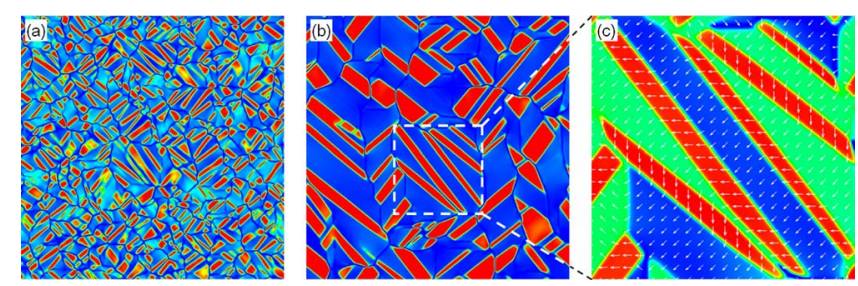

FIG. 1. (Color online) Computer simulation of coherent microstructures of phase decomposition in PZT near MPB. (a) Two-phase morphology at earlystage decomposition (simulation time $t=1$ ) and (b) coarsened morphology $(t=30)$, where color scale shows the composition profile: tetragonal phase in red $(x \approx 0.77)$ and rhombohedral phase in blue $(x \approx 0.38)$. (c) Close-up visualization of a portion of the phase-coexisting multidomain microstructure shown in (b), where arrows represent the in-plane components $\left(P_{x}, P_{y}\right)$ of polarization vector and the out-of-plane component $\left(P_{z}\right)$ is represented by color: red domains $\left(P_{z}=0\right)$ are tetragonal phase, green $\left(P_{z}>0\right)$ and blue $\left(P_{z}<0\right)$ domains are rhombohedral phase.

energy parameters, ${ }^{2}$ ultrasonically measured elastic constants, ${ }^{17}$ and the Rietveld refinement-derived electrostriction coefficients ${ }^{18}$ for PZT. A value of $w=0.5 k_{B} T_{C} / \Omega$ is used for the positive enthalpy of mixing. ${ }^{2,12}$ The simulation is performed with $512 \times 512$ computational cell with grid size of $\sim 2 \mathrm{~nm}$.

The computer simulation reveals characteristic multidomain microstructures, where lamellar domains of tetragonal and rhombohedral phases coherently coexist with welldefined crystallographic orientation relationships, as shown in Fig. 1. We consider PZT system at room temperature with nominal MPB composition, $x=x_{m}=0.5$ defined in Eq. (2). ${ }^{2}$ Figures 1(a) and 1(b) show the simulated two-phase microstructural morphology at early-stage decomposition and after coarsening, respectively. The two representative stages of coherent phase decomposition exhibit essentially the same morphological characteristics, despite that the phases have not fully decomposed in Fig. 1(a) and the microstructure coarsens with time in Fig. 1(b). Compared with conventional phase decompositions, the ferroelectric phase decomposition shows some peculiar microstructural features from its very early stage, which result from the coherency strain and, in particular, the electrostatics of polarization. As shown in Fig. 1 , the interfaces of lamellar compositional domains of tetragonal (red) phase (i.e., the habit planes of the lamellas) exhibit well-defined orientations, which are close to $\{340\}$ in twodimensional simulation. Analysis shows that such habit planes minimize the interfacial charge density by satisfying $\left(\mathbf{P}^{R}-\mathbf{P}^{T}\right) \cdot \mathbf{n} \approx 0$, where $\mathbf{n}$ is the interface normal vector, and $\mathbf{P}^{R}$ and $\mathbf{P}^{T}$ are the polarization vectors of the decomposed rhombohedral and tetragonal phases, respectively. Figure 1 (c) shows a close-up view of the phase-coexisting multidomain microstructure. The $\{340\}$ interfaces are $55^{\circ}$ domain walls between rhombohedral and tetragonal phases. The domains of tetragonal (red) phase mutually form twins of $\{110\}$ twin boundaries, which are $90^{\circ}$ ferroelectric domain walls; the domains of rhombohedral (green and blue) phase form twins of either $\{100\}$ or $\{110\}$ twin boundaries, which are $109^{\circ}$ and $71^{\circ}$ domain walls, respectively. These twins are in agreement with the crystallographic analysis of domain microstructures. Another peculiar behavior of ferroelectric phase decomposition is the Ti depletion along ferroelectric domain walls, i.e., composition $x$ is a little lower along domain walls than inside domains, which is visible as deep blue narrow zones running along tetragonal-rhombohedral interfaces and inside rhombohedral (blue) phase in Fig. 1(b). The 
domain wall $\mathrm{Ti}$ depletion results from the modification of diffusional decomposition by the local stress and electric fields in domain wall regions.

The computer simulation shows that coherent phase decomposition in PZT produces nanoscale multidomain microstructures. Experimental investigation ${ }^{5}$ and theoretical estimation $^{2}$ also show that the diffusion rate in PZT produces composition heterogeneities with a typical wavelength of the order of $10 \mathrm{~nm}$. This domain size is significantly below the coherence length of diffraction radiations used to characterize PZT phases. As a result, coherent diffraction effects must be taken into account in the interpretation of diffraction data. Nanotwin diffraction analysis shows that diffraction perceives nanotwins of tetragonal and rhombohedral phases as monoclinic phases. ${ }^{9}$ Such coherent diffraction effect of nanodomains has recently been experimentally confirmed in PZT. ${ }^{10}$ Clearly, without taking into account the nanoscale domain microstructures, the diffraction data could be easily misinterpreted. Therefore, it is important to gain insight into the coherent diffraction effect of the phase-coexisting multidomain microstructures shown in Fig. 1. It is worth noting that the monoclinic lattice corresponds to the average symmetry of the nanotwins and exhibits intrinsic lattice parameter relationships, ${ }^{19,20}$ as predicted by both diffraction analysis ${ }^{9}$ and crystallographic analysis through nanodomain averaging. ${ }^{19,20}$ Following nanodomain averaging, the phasecoexisting multidomain microstructure shown in Fig. 1 exhibits, in general, an averaged crystal structure of pseudoorthorhombic symmetry with small triclinic shear distortions. When lamellar domains of one tetragonal orientation variant dominate in a local volume of coherent diffraction, the averaged lattice has a pseudotetragonal symmetry with lattice parameters $c=\omega c_{t}+(1-\omega) a_{r}$ and $a=\omega a_{t}+(1-\omega) a_{r}$, where $c_{t}$, $a_{t}$, and $a_{r}$ are the lattice parameters of tetragonal and rhombohedral phases, respectively, and $\omega$ is the volume fraction of tetragonal phase. These relations show that the measured lattice parameters $c$ and $a$, as assigned to tetragonal phase, strongly depend on the volume fraction $\omega$ of tetragonal phase. With the nominal composition $x$ of PZT samples decreasing from tetragonal to rhombohedral phase fields across the MPB, $\omega$ rapidly drops from 1 to 0 over a narrow composition range, whose width depends on both the positive enthalpy of mixing and diffusion rate. Accompanying $\omega$ change, the measured tetragonal lattice parameters $c$ and $a$ rapidly converge toward $a_{r}$ with tetragonality ratio $c / a$ rapidly decreasing to 1 . Such predictions are, in fact, consistent with the Rietveld refinement-measured PZT lattice parameters, where $c$ and $a$ change smoothly with composition before rapidly converging toward $a_{r}$ within a narrow composition range of MPB phase coexistence. ${ }^{18}$ It, thus, raises the question whether such a rapid lattice parameter variation near MPB is an intrinsic behavior or instead a result of coherent diffraction effect due to the phase-coexisting nan- odomain microstructure that was undetected in previous conventional interpretation of diffraction data. Answers to this question will help better understand the nature of MPB, phase coexistence, PZT phase diagram, and enhanced piezoelectricity near MPB. To further address this important issue, detailed computational diffraction analysis of the phasecoexisting multidomain microstructures (Fig. 1) is underway.

In summary, computer modeling and simulation of coherent phase decomposition in PZT of MPB composition reveals characteristic phase-coexisting multidomain microstructures, where nanoscale lamellar domains of tetragonal and rhombohedral phases coexist with well-defined crystallographic orientation relationships. Diffraction experiments only detect the domain-averaged structural information. Knowledge of such nanodomain microstructures is crucial for correctly interpreting diffraction data, identifying MPB phases, understanding phase coexistence, and constructing phase diagram.

The parallel computer simulations were performed on Data Star at San Diego Supercomputer Center and System X at Virginia Tech.

${ }^{1}$ B. Jaffe, W. R. Cook, and H. Jaffe, Piezoelectric Ceramics (Academic, London, 1971).

${ }^{2}$ G. A. Rossetti, Jr., W. Zhang, and A. G. Khachaturyan, Appl. Phys. Lett. 88, 072912 (2006).

${ }^{3}$ V. A. Isupov, Sov. Phys. Solid State 10, 989 (1968) [Fiz. Tverd. Tela (Leningrad) 10, 1244 (1968)]; Phys. Solid State 43, 2262 (2001) [Fiz. Tverd. Tela (Leningrad) 43, 2171 (2001)]; Ferroelectrics 266, 91 (2002).

${ }^{4}$ L. Benguigui, Solid State Commun. 11, 825 (1972); P. Ari-Gur and L. Benguigui, ibid. 15, 1077 (1974).

${ }^{5}$ K. Kakegawa, J. Mohri, T. Takahashi, H. Yamamura, and S. Shirasaki, Solid State Commun. 24, 769 (1977); K. Kakegawa, J. Mohri, S. Shirasaki, and K. Takahashi, J. Am. Ceram. Soc. 65, 515 (1982).

${ }^{6}$ W. Cao and L. E. Cross, Phys. Rev. B 47, 4825 (1993).

${ }^{7}$ A. M. Glazer, P. A. Thomas, K. Z. Baba-Kishi, G. K. H. Pang, and C. W. Tai, Phys. Rev. B 70, 184123 (2004).

${ }^{8}$ B. Noheda, D. E. Cox, G. Shirane, J. A. Gonzalo, L. E. Cross, and S. E. Park, Appl. Phys. Lett. 74, 2059 (1999).

${ }^{9}$ Y. U. Wang, Phys. Rev. B 74, 104109 (2006); 76, 024108 (2007).

${ }^{10}$ K. A. Schönau, L. A. Schmitt, M. Knapp, H. Fuess, R. A. Eichel, H. Kungl, and M. J. Hoffmann, Phys. Rev. B 75, 184117 (2007).

${ }^{11}$ A. F. Devonshire, Philos. Mag. 40, 1040 (1949).

${ }^{12}$ M. V. Rane, A. Navrotsky, and G. A. Rossetti, Jr., J. Solid State Chem. 161, 402 (2001).

${ }^{13}$ S. Semenovskaya and A. G. Khachaturyan, J. Appl. Phys. 83, 5125 (1998).

${ }^{14}$ Y. L. Li, S. Y. Hu, Z. K. Liu, and L. Q. Chen, Appl. Phys. Lett. 78, 3878 (2001).

${ }^{15}$ W. F. Rao and Y. U. Wang, Appl. Phys. Lett. 90, 041915 (2007); 90, 182906 (2007).

${ }^{16}$ J. W. Cahn and J. E. Hilliard, J. Chem. Phys. 28, 258 (1958).

${ }^{17}$ T. Fett and D. Munz, J. Test. Eval. 28, 27 (2000).

${ }^{18}$ W. F. Rao and Y. U. Wang (unpublished).

${ }^{19}$ Y. M. Jin, Y. U. Wang, A. G. Khachaturyan, J. F. Li, and D. Viehland, Phys. Rev. Lett. 91, 197601 (2003); J. Appl. Phys. 94, 3629 (2003).

${ }^{20}$ Y. U. Wang, Phys. Rev. B 73, 014113 (2006). 